\title{
Variations in tooth preparations for resin-bonded all-ceramic crowns in general dental practice
}

\author{
A. F. Sutton' and J. F. McCord ${ }^{2}$
}

Objective To investigate variations in tooth preparations for resin-bonded all-ceramic crowns (RBCs) in general dental practice (GDP).

Design Laboratory-based retrospective analysis of dies for RBCs. Setting General dental practices in the UK and Ireland (2000). Methods A sample $(n=132)$ of laboratory models containing 180 tooth preparations for RBCs, featuring work from different general dental practitioners was obtained from four commercial dental laboratories. Aspects of the preparations were quantified and compared with accepted criteria defined following a review of the literature.

Results The teeth found to be most frequently prepared for RBCs were maxillary incisors (41\%). Margin positions were variably positioned with $29 \%$ of the preparations on the buccal aspect having subgingival margins. There were many tooth preparation dies for low fusing RBCs (47\%) and Chameleon Fortress RBCs $(62 \%)$ demonstrating overpreparation in the mesiodistal plane. The majority of the margins ( $84 \%$ buccally and $79 \%$ lingually) of the dies examined exhibited appropriate shoulder or chamfer finishes. Of the Chameleon Fortress preparations analysed, 86\% had been underprepared occlusally. $42 \%$ of the teeth had been prepared with no regard to tooth morphology and demonstrated just one plane of reduction. The majority $(93 \%)$ of the clinicians failed to provide any information regarding the shade of the prepared tooth stump.

Conclusions On the evidence of this survey of this sample of general dental practitioners' work, it was found that relevant guidelines for the preparations of RBCs are not being fully adhered to.

$\mathrm{B}$ owen first described bonding to dentine in 1965, and from this, modern dentine-bonding materials have been developed, principally over the past 15 years. ${ }^{1}$ A by-product of this research extended the indications for all-porcelain restorations. Demand for a more pleasing appearance of dental restoration and public fears about adverse side effects of dental metals and alloys have led to the increased use of ceramics.

The resin-bonded all-ceramic crown (RBC) is a full-coverage restoration which is bonded to underlying enamel or dentine using a dentine bonding system in conjunction with a dual-cure resin com-

${ }^{1}$ Specialist Registrar, Restorative Dentistry, Liverpool University Dental Hospital;

${ }^{2}$ Head of the Unit of Prosthodontics, Unit of Prosthodontics, Department of

Restorative Dentistry, Turner Dental School, University Dental Hospital of

Manchester, Higher Cambridge Street, Manchester M15 6FH

${ }^{*}$ Correspondence to: Finlay Sutton, Liverpool University Dental Hospital,

Pembroke Place, Liverpool L3 5PS

email: Finlaysutton@another.com

REFEREED PAPER

Received 18.12.00; Accepted 21.03.01

(C) British Dental Journal 2001; 191: 677-681 posite luting material. It was first described by Rezinkor in 1987 and named a dentine-bonded all-ceramic crown. ${ }^{2}$

Crothers et al. defined the RBC as a porcelain veneer which has been extended circumferentially to involve a substantial proportion of the lingual/palatal aspect of the tooth. ${ }^{3}$

The strength of the final restoration is thought to result from a synergism of bonding of the various component parts, namely, the underlying tooth, the etched ceramic restoration and the resin composite lute. $^{4}$

The ceramic materials appropriate for the construction of RBCs are: ${ }^{5}$

- Low fusing porcelains

- Leucite reinforced porcelains

- Pressed glass ceramics

Each of the porcelain systems suitable for RBCs have subtle differences in preparation design recommendations, depending on the type of ceramics used in their fabrication. The universal guidelines for all RBCs are the need for rounded internal edges and angles, the placement of the restoration margin either level with or above the gingival margin and the communication of intrinsic staining of the prepared tooth stump to the technician. ${ }^{6}$

Table 1 presents RBC tooth preparation guidelines for low fusing porcelains, leucite reinforced porcelains (Fortress Chameleon Dental Products, information leaflet, Kansas City, KN, USA) and pressed glass ceramics (Ivoclar-Vivadent, IPS Empress information leaflet, Schaan, Liechtenstein). $5,7,8$

Clinical guidelines, techniques and types of preparations for RBCs are well described in the literature, however there is no real in-vivo evidence base to them. Nevertheless, to date, no study has investigated the extent to which these guidelines are employed in

Table I. Specific guidelines for RBCs

Type of RBC Specific guidelines

Low fusing porcelains

Chamfer or shoulder of $0.4-0.6 \mathrm{~mm}$

Occlusal and/or incisal reduction of Imm minimum

Discoloration of the prepared tooth should be indicated to the technician

Leucite reinforced porcelain

A minimum $0.3 \mathrm{~mm}$ chamfer or butt margin $2.5 \mathrm{~mm}$ occlusal reduction allowed for working cusps

Severe discolouration of the underlying natural tooth surface should be indicated

Pressed glass ceramics

Circular chamfer or shoulder of I mm Incisal third of tooth reduction of $1.5 \mathrm{~mm}$ Occlusal and/or incisal reduction of $2 \mathrm{~mm}$ Supply of shade of prepared tooth stump 
Fig. I a Anatomically (two or more planes) prepared upper incisor tooth on the labial aspect

Fig. I b Non-anatomically (one-plane) prepared upper incisor tooth on the labial aspect
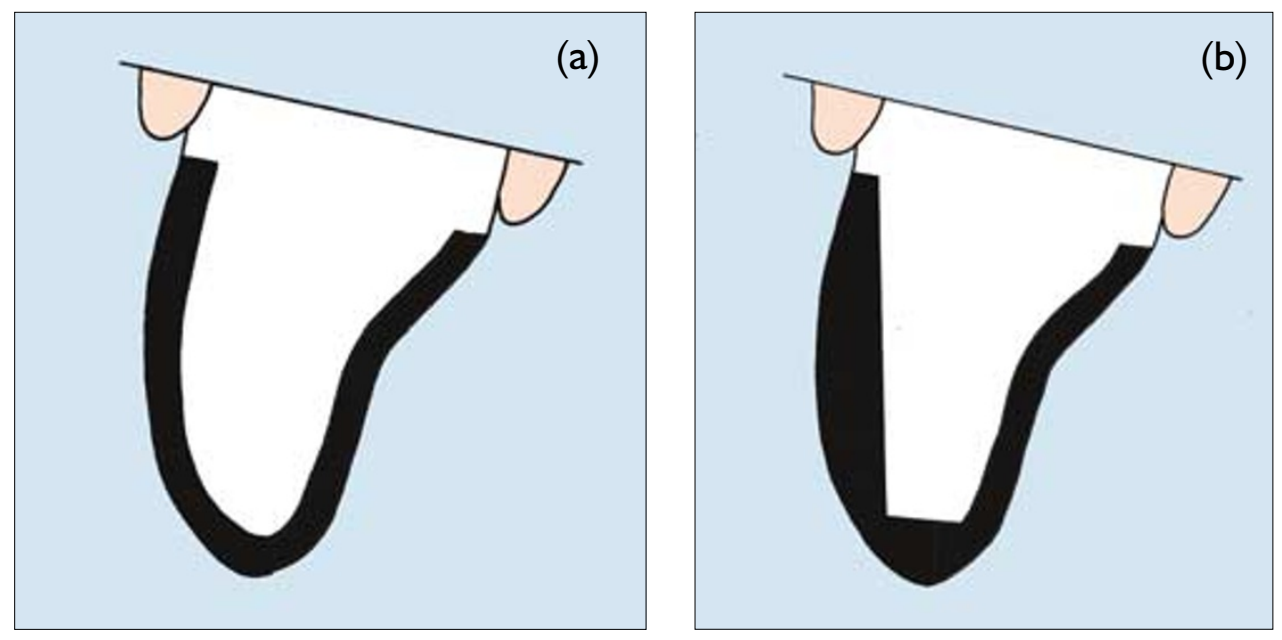

general dental practice. The aim of this study, therefore, was to investigate variations in tooth preparation designs for RBCs provided by GDPs. Burke et al. have stated that the tooth preparation design for these types of restoration can influence the stability, durability, aesthetics and accuracy of fit of the restoration. ${ }^{9}$ The study consisted of examining $180 \mathrm{RBC}$ working dies, analysing the form of the preparations and thereby determining if current guidelines are being followed.

\section{Materials and methods}

Four commercial dental laboratories in England that provide these types of restoration for general practitioners in the UK and Ireland agreed to participate in the study. The laboratories were selected because of the their long-term experience fabricating RBCs and the high volume of production.

Each laboratory provided the following:

- A series of duplicate casts of working models containing one or more RBC tooth preparation dies

- A duplicate cast of the opposing model

- A copy of the laboratory prescription form containing the patient's name and the dentist's name

The sample was drawn by selecting consecutive cases until 180 dies were gathered from 132 working casts. All of the 132 casts contained different clinicians' work. The sample size was such that it exposed most of the dentists regularly requesting RBCs. The sample split between the laboratories was 67, 42, 39 and 32. There was no attempt to differentiate between work provided under NHS or private contract and the laboratories were not weighted. For each case the following information was recorded:

- Number of RBCs required

- Tooth/teeth prepared

- The ceramic material requested

- The tooth preparation margin position in relation to the gingival margin position on the buccal/labial aspect

- The tooth preparation margin position in relation to the gingival margin position on the lingual/palatal aspect

- The total amount of tooth reduction in the buccolingual plane of the preparation

- The total amount of tooth reduction in the mesiodistal plane of the preparation

- The buccal/labial margin design of the tooth preparation

- The lingual/palatal margin design of the tooth preparation

- The occlusal reduction of the tooth preparation

- The buccal/labial planes of the tooth preparation

- The presence of a supplied shade of the prepared tooth stump by the GDP
The positions of the margins of the preparation relative to the gingival margins were recorded using the scoring system described by Brunton and Wilson. ${ }^{10}$ As a convention, the location of the principal portion of the margin determined the score. Assessment of the dies was carried out prior to the dies being trimmed with the aid of a Williams periodontal probe (Ash, UK). The use of gingival retraction was not taken into account.

The total amount of tooth reduction in the buccolingual and mesiodistal planes of the preparation measurement was only possible if the corresponding contralateral tooth was present and unrestored. Seventy-nine dies out of 180 were suitable for this measurement. Mayers et al. ${ }^{11}$ have shown that contralateral teeth are directly comparable in dimension. The measurement was made using dial vernier callipers (Mitutoyo, Japan) with thinly machined measuring tips. The measurement recordings of the prepared teeth were made with the calliper in the buccolingual and mesiodistal planes. The antimeres were then measured in the same planes. The total amount of preparation reduction was then calculated by deducting the unprepared contralateral tooth width in the two planes, from the width of the prepared teeth. This gave a total amount of tooth reduction and not the depth of reduction on each axial wall. The preparation margin design was assessed visually with the aid of x2.5 magnification (Dental Loupes, Orascoptic, USA).

The occlusal clearance was assessed using a method described by Etemadi et al. ${ }^{12}$ This was measured by adapting silicone laboratory impression putty (Indurent putty, Zhermack SpA, Italy) onto the tooth preparation and then positioning the opposing cast into maximum intercuspation. The articulation of the casts was performed by hand, as it has been demonstated that this is the most reproducible and accurate method of articulating in maximum intercuspation, when there are adequate numbers of contacts around both arches. ${ }^{13}$ Once the putty had hardened, it was removed from the casts and trimmed. A dial micrometer (Brascon Ltd, UK) was then used to measure the distance between the preparation and the opposing tooth. The smallest of three measurements recorded were collated for analysis.

To assess the plane or planes of tooth reduction, each die was axially sectioned through the midline with a carborundum disc (thickness $0.5 \mathrm{~mm}$ ). The preparations were collected into three groups; namely those that had been prepared in one plane (nonanatomically prepared), those prepared in two or three planes (anatomically prepared) (Fig. 1) and those that showed no sign of preparation.

To investigate the inter- and intra-examiner reproducibility of the scoring systems, a random subsample of dies $(n=40)$ was selected and re-scored by the examiners after 7 days and the results compared. Kappa statistics were used to quantify and analyze the inter- and intra-examiner reliability. ${ }^{14}$ 


\section{Results}

The majority of cases investigated were for one RBC only (73\%), with maxillary central incisors most frequently prepared (41\%). Of the dies examined, 143 (79\%) were of maxillary teeth.

Table 2 shows the results of the tooth preparation margin positions in relation to the gingival margin position on the buccal/labial and lingual/palatal aspect. Of the $180 \mathrm{RBC}$ tooth preparation dies examined on the buccal and labial aspects $72 \%$ demonstrated margin positions supragingival or level with the gingival margin; $22 \%$ had subgingival margins and $6 \%$ demonstrated no clear margin.

Table 3 shows the total amount of tooth tissue reduction in the buccolingual and mesiodistal planes of the RBC tooth preparations. In total $32 \%$ of the tooth preparation dies demonstrated overpreparation, 54\% exhibited appropriate depth of preparation and 14\% (all Empress) showed underpreparation.

Table 4 lists the distribution of the buccal/labial and lingual/ palatal margin designs for the RBC tooth preparations examined: $82 \%$ demonstrated either a shoulder or chamfer margin design; $13 \%$ and $6 \%$ had either a feathered or no clear margin design respectively.

The occlusal reduction measurements are listed in Table 5:27\% of the low fusing porcelain, $86 \%$ of the Chameleon Fortress and $36 \%$ of the Empress tooth preparation dies demonstrated underpreparation occlusally.

The buccal/labial planes of the preparations, as recorded from the casts, are listed in Table 6. Seven of the 180 dies examined were for post crowns with RBCs and therefore the buccal planes of preparation could not be assessed.

None of the tooth preparations for low fusing RBCs, $2 \%$ of the Chameleon Fortress RBCs and 23\% of the Empress RBCs was sup- plied with an indication of the intrinsic staining of the underlying tooth structure.

The Kappa statistics quantifying the intra-examiner and interexaminer variability for the various measurements performed ranged from 0.73 to 1.00 and 0.69 to 0.94 respectively.

\section{Discussion}

This study investigated variations in the form of tooth preparations for resin-bonded all-ceramic crowns in general dental practice.

From 132 working models, 180 dies were included and this number allowed meaningful analysis of the results with a diverse cross section of clinicians' work. The Kappa statistics quantifying the intra-examiner reliability ranged from 0.73 to 1.00 and the interexaminer reliability ranged from 0.69 to 0.94 indicating strength of agreement from substantial to almost perfect. ${ }^{14}$

Other workers' studies in general dental practice have employed this method of commercial laboratory based retrospective analysis, although this merely samples an undefined percentage of the dental population. ${ }^{10,15,16}$

A significant proportion of dies included in the sample (41\%) were of maxillary central incisors. This corresponds to the findings of Brunton and Wilson in their study of porcelain veneer preparations, where a significant proportion included in the sample were of maxillary incisors. ${ }^{10}$ It is often assumed that patients tend to be more concerned with unsightly maxillary rather than mandibular teeth, mandibular teeth being less visible in function. ${ }^{17}$ This was supported by the findings that $143(79 \%)$ dies of maxillary teeth were included in the sample.

Table 2. Results of the tooth preparation margin positions in relation to the gingival margin positions on the buccal and lingual aspects (figures in italics are in accordance with current guidelines)

\begin{tabular}{|c|c|c|c|c|c|c|c|}
\hline & \multicolumn{6}{|c|}{ Buccal and lingual margin positions } \\
\hline & & $\begin{array}{l}>2 \mathrm{~mm} \\
\text { supragingival }\end{array}$ & $\begin{array}{l}\quad \leq 2 \mathrm{~mm} \\
\text { supragingival }\end{array}$ & $\begin{array}{l}\text { Level with } \\
\text { the gingival margin }\end{array}$ & Subgingival & $\begin{array}{l}\text { No clear } \\
\text { margin }\end{array}$ & Total \\
\hline \multirow[t]{3}{*}{ Type of RBC } & Low Fusing & $6(4 \%)$ & 65 (43\%) & 47 (3І\%) & $30(20 \%)$ & $4(3 \%)$ & $152(100 \%)$ \\
\hline & Chameleon Fortress & $7(7 \%)$ & $37(36 \%)$ & 31 (30\%) & $16(16 \%)$ & II (II\%) & $102(100 \%)$ \\
\hline & Empress I \& II & $3(3 \%)$ & 32 (30\%) & 32 (30\%) & $32(30 \%)$ & $7(7 \%)$ & $106(100 \%)$ \\
\hline Total & & $16(4 \%)$ & 134 (37\%) & $110(31 \%)$ & $78(22 \%)$ & $22(6 \%)$ & $360(100 \%)$ \\
\hline
\end{tabular}

Table 3. Results of the total amount of tooth reduction in the buccolingual and mesiodistal planes of the tooth preparations (figures in italics are in accordance with current guidelines)

\begin{tabular}{|c|c|c|c|c|c|c|}
\hline & & \multicolumn{5}{|c|}{ Total buccolingual and mesiodistal tooth reduction } \\
\hline & & $>3 \mathrm{~mm}$ & $\leq 3 \mathrm{~mm}>2 \mathrm{~mm}$ & $\leq 2 \mathrm{~mm}>1 \mathrm{~mm}$ & $\leq \mathrm{Imm} \& 0 \mathrm{~mm}$ & Total \\
\hline Type of RBC & $\begin{array}{l}\text { Low Fusing } \\
\text { Chameleon Fortress } \\
\text { Empress I \& II }\end{array}$ & $\begin{array}{l}7(11 \%) \\
4(10 \%) \\
5(10 \%)\end{array}$ & $\begin{array}{l}16(25 \%) \\
19(45 \%) \\
25(48 \%)\end{array}$ & $\begin{array}{l}28(44 \%) \\
13(3 / \%) \\
21(40 \%)\end{array}$ & $\begin{array}{r}13(20 \%) \\
6(14 \%) \\
1(2 \%)\end{array}$ & $\begin{array}{l}64(100 \%) \\
42(100 \%) \\
52(100 \%)\end{array}$ \\
\hline Total & & $16(10 \%)$ & $60(38 \%)$ & $62(39 \%)$ & $20(13 \%)$ & $158(100 \%)$ \\
\hline
\end{tabular}

Table 4. Results of the buccal and lingual margin designs of the RBC tooth preparations (figures in italics are in accordance with current guidelines)

\begin{tabular}{|c|c|c|c|c|c|c|}
\hline & & \multicolumn{5}{|c|}{ Buccal and lingual margin designs } \\
\hline & & Shoulder & Chamfer & Feathered & No clear margin & Total \\
\hline Type of RBC & $\begin{array}{l}\text { Low Fusing } \\
\text { Chameleon Fortress } \\
\text { Empress I \& II }\end{array}$ & $\begin{array}{l}67(44 \%) \\
50(49 \%) \\
72(68 \%)\end{array}$ & $\begin{array}{l}65(43 \%) \\
23(23 \%) \\
16(15 \%)\end{array}$ & $\begin{array}{r}16(11 \%) \\
19(19 \%) \\
10(9 \%)\end{array}$ & $\begin{array}{r}4(26 \%) \\
10(10 \%) \\
8(8 \%)\end{array}$ & $\begin{array}{l}152(100 \%) \\
102(100 \%) \\
106(100 \%)\end{array}$ \\
\hline Total & & $189(53 \%)$ & 104 (29\%) & $45(13 \%)$ & $22(6 \%)$ & $360(100 \%)$ \\
\hline
\end{tabular}


Table 5. Results of the occlusal reduction of the RBC tooth preparations. (figures in italics are in accordance with current guidelines)

\begin{tabular}{|c|c|c|c|c|c|c|}
\hline & & \multicolumn{4}{|c|}{ Occlusal reduction } & \multirow[b]{2}{*}{ Total } \\
\hline & & $>3 \mathrm{~mm}$ & $\leq 3 \mathrm{~mm}>2 \mathrm{~mm}$ & $\leq 2 \mathrm{~mm}>1 \mathrm{~mm}$ & $\leq \mathrm{Imm} \& 0 \mathrm{~mm}$ & \\
\hline Type of RBC & $\begin{array}{l}\text { Low Fusing } \\
\text { Chameleon Fortress } \\
\text { Empress I \& II }\end{array}$ & $\begin{array}{l}8(12 \%) \\
1 \quad(2 \%) \\
6(18 \%)\end{array}$ & $\begin{array}{r}15(23 \%) \\
5(11 \%) \\
6(18 \%)\end{array}$ & $\begin{array}{r}25(38 \%) \\
23(52 \%) \\
9(27 \%)\end{array}$ & $\begin{array}{l}18(27 \%) \\
15(34 \%) \\
12(36 \%)\end{array}$ & $\begin{array}{l}66(100 \%) \\
44(100 \%) \\
33(100 \%)\end{array}$ \\
\hline Total & & $15(10 \%)$ & $26(18 \%)$ & 57 (40\%) & 45 (32\%) & $143(100 \%)$ \\
\hline
\end{tabular}

Table 6 Results of the buccal/labial planes of the RBC tooth preparations. (figures in italics are in accordance with current guidelines)

\begin{tabular}{llllll}
\hline & & \multicolumn{2}{c}{ Buccal planes of preparation } \\
\cline { 3 - 6 } & & $\begin{array}{c}\text { Anatomic } \\
\leq 2 \text { planes }\end{array}$ & $\begin{array}{c}\text { Non-anatomic } \\
\text { I plane }\end{array}$ & $\begin{array}{c}\text { No sign of } \\
\text { preparation }\end{array}$ \\
\hline Type of RBC & Low Fusing & & & & Total \\
& Chameleon Fortress & $25(50 \%)$ & $25(37 \%)$ & $1(1 \%)$ & $75(100 \%)$ \\
\hline Total & Empress I \& II & $28(58 \%)$ & $20(42 \%)$ & $0(0 \%)$ & $50(100 \%)$ \\
\hline
\end{tabular}

It was perhaps worrying to note that a considerable proportion of the tooth preparations (29\%) had subgingival margins on the buccal aspect. This value is similar to those of previous veneer studies where Brunton and Wilson found $20 \%$ and Karlsson et al. found $32 \%$ of veneer preparations possessed subgingival margins. ${ }^{10,18}$ The subgingival margin placement of RBCs is of importance considering that there is greater possibility of microleakage if the margins are placed on dentine and cementum. ${ }^{19,20}$ The bonding of the luting material will be jeopardised if moisture control is inadequate, which is the case in subgingival preparations. ${ }^{4}$ However, there are valid aesthetic reasons for preparing teeth beneath the free margin of the gingivae, such as the presence of an existing restoration and the cosmetic demands of the patient. ${ }^{21}$ In such circumstances it may be prudent to think in terms of a conventional crown. ${ }^{2}$

Seventy-nine (44\%) samples were suitable for analysing the total reduction in the buccolingual and mesiodistal planes, the remaining were not used because of lack of an unrestored antimere tooth. The method used to measure the total amount of tooth reduction merely took into account the total amount of tooth reduction in one plane and not the individual axial wall preparation depth, therefore one aspect of the tooth may have been appropriately prepared, whilst the other may have been incorrect. However, the measurements still served as a guide for axial wall tooth reduction.

For the low fusing porcelain RBCs (47\%) and Chameleon Fortress RBCs (62\%) there was a tendency to overprepare the teeth in the mesiodistal plane. Forty-eight per cent of the Chameleon Fortress RBCs tooth preparations were overprepared in the buccolingual plane. Overpreparation of the teeth negates the advantages that RBCs demonstrate and may lead to loss of pulpal vitality. Periradicular pathology in conventionally crowned teeth has been clearly demonstrated in the studies by Saunders and Saunders, ${ }^{22}$ and Christensen. ${ }^{23}$ Overpreparation can also reduce the amount of enamel remaining for bonding, leaving the restoration dependent on dentine bonding for retention and strength. ${ }^{4}$ Total tooth reduction in excess of $2 \mathrm{~mm}$ may leave unsupported porcelain, which as stated by Crothers et al. is vulnerable to fracture. ${ }^{2}$

The results of this study indicated that of the Empress crown preparations, $54 \%$ in the buccolingual plane and $31 \%$ in the mesiodistal plane had been underprepared. However, in a recent in-vitro study by Burke, it was demonstrated that the fracture strength of IPS Empress crowns with marginal reduction of
$0.5 \mathrm{~mm}$ and bonded with the $3 \mathrm{M}$ dentine bonding system (3M, St Paul, MN, USA) was higher than natural unrestored teeth. ${ }^{24}$ Therefore, minimal axial wall reduction $(0.5 \mathrm{~mm})$ may be clinically appropriate.

With regard to the marginal design, $84 \%$ of the buccal and $79 \%$ of the lingual margins of the dies analysed had shoulder or chamfer preparations. Somewhat worryingly however, the remaining $16 \%$ on the buccal and $21 \%$ on the lingual aspects of the dies demonstrated a feathered margin design or no detectable margin. In these cases at cementation, the RBC may have a tendency to fracture at the thin margin. The technician, in an attempt to strengthen the margin, may overbuild the RBC, which may result in a bulbous margin with plaque retention leading to periodontal problems and/or an unsightly emergence profile.

In total, 143 dies were measured for occlusal reduction. The remaining 37 were not used either because of the lack of sufficient numbers of occlusal contacts, leaving it impossible to accurately hand articulate in maximum intercuspation, or lack of opposing tooth units.

The recommended amount of occlusal tooth reduction varies depending on the type of porcelain system used. Twenty seven per cent of the tooth preparations for low fusing RBCs, $86 \%$ of the Chameleon Fortress and 36\% of the Empress RBCs demonstrated underpreparation occlusally. It is important to provide an adequate bulk of porcelain in areas exposed to heavy loading. ${ }^{2}$ It has been stated that the ultimate strength of a material is dependent on its thickness. Porcelain responds in accordance with the engineering Law of Beams which states that doubling the thickness of a material increases its strength four-fold. ${ }^{25}$

Conventional wisdom would apply recommendations that teeth are prepared for crowns and veneers according to the morphology of the tooth. ${ }^{17}$ It is difficult to achieve an appropriate depth of preparation in the cervical third of the tooth if the tooth is prepared with no regard to anatomic form. ${ }^{14}$ The majority of teeth (56\%) were found to have been prepared with due regard to tooth morphology. Forty-two per cent were prepared with only one plane of preparation on the buccal/labial aspect. This may result in a preparation with an overcontoured or 'bulky' restoration in the cervical third and this will almost certainly result in periodontal problems unless oral hygiene standards are exceptionally high. Conversely non-anatomical preparation of the tooth may produce 
adequate cervical reduction, but produce under-reduction incisally. In addition, anatomical tooth preparation is of importance with respect to increasing the retention and resistance form of the preparation. ${ }^{17}$ Poor contour of the restoration may in addition result in an unaesthetic restoration since the eye's perception of tooth form is of a higher order than tooth shade. ${ }^{26}$ One $(0.5 \%)$ of the teeth observed demonstrated no sign of preparation. The contour of a restoration placed in this instance would unquestionably be poor.

None of the tooth preparations for low fusing RBCs, $2 \%$ of the Chameleon Fortress RBCs and 23\% of the Empress RBCs were supplied with an indication of the degree of discolouration of the underlying tooth structure. In the authors' opinion, from the 180 tooth preparations examined it must be expected that more than 12 exhibited intrinsic discolouration. It is recommended by Crothers et al. for RBCs, and Cassidy et al. for veneers that discolouration of the underlying tooth structure is supplied to the technician to mask the discolouration with opaque porcelain or to place extra die spacer to allow a thicker amount of opaque resin for bonding. ${ }^{2,6}$ Ivoclar-Vivadent supply a shade guide specifically for the prepared tooth stump for use with the IPS Empress system. The aesthetic results of these crowns would be improved if more clinicians communicated this shade to the technician.

From the results presented in this paper, it has been shown that there are wide variations in the preparations for resin-bonded allceramic crowns in general dental practice.

On the evidence of this survey of clinicians' work in general dental practice, it was found that relevant guidelines for the preparations of RBCs are not being entirely adhered to.

\section{Conclusion}

This study has shown that preparations for RBCs of the clinicians' work examined may be found to vary widely. The following conclusions may be drawn:

- There was a tendency for the margins of the tooth preparations to be placed subgingivally.

- There were a significant number of the low fusing RBCs and Chameleon Fortress RBCs dies which had been overprepared.

- The majority of the margins of the tooth preparation dies examined, correctly demonstrated either shoulder or chamfer finishes. However, there were a number with either no detectable margin or feathered margins.

- Many of the tooth preparations analysed had been underprepared occlusally.

- More than half of the teeth had been prepared with no regard to tooth morphology, demonstrating just one plane of reduction.

- The majority of the prescribing practitioners failed to provide any information regarding the shade of the prepared tooth stump.

\section{Recommendations for further research}

- Clinical trials are needed to determine the long-term durability of RBCs.

- In-vivo and in-vitro clinical trials to determine the optimum combination of porcelain, resin cement, dentine bonding agent and tooth preparation design.

- More long-term studies in general dental practice are necessary, where the majority of the RBC restorations are placed.
This type of restoration has been in use for less than ten years and therefore is a relatively recent addition to the dentists' armamentarium. Postgraduate education for general dental practitioners who were not trained to use RBCs as undergraduates is probably necessary to improve the knowledge of the required preparation designs.

1 Bowen R L. Adhesive bonding of various materials to hard tooth tissues. Part 2: bonding to dentin promoted surface-active comonomer. J Dent Res 1965; 44: 895-902.

2 Rezinkor H. The dentin bonded porcelain crown. In: Clinical procedures manual. Cedar Rapids: Dental Prosthetic Services Inc., 1987.

3 Crothers A J R, Wassell R W, Allen P F. The resin-bonded porcelain crown: A rationale for use on anterior teeth. Dent Update 1993; 20: 388-395.

4 Burke F J T, Qualtrough A J E, Hale R W. Dentine-bonded all-ceramic crowns: current status. J Am Dent Assoc 1998; 129: 455-460.

5 Burke F J T, Watts D C. Fracture resistance of teeth restored with dentinbonded crowns. Quintessence Int 1994; 25: 335-340.

6 Cassidy M, McLaughlin W S, Grey N J. Aesthetics and porcelain veneers. Restorative Dent 1989; 5: 42-45.

7 Chameleon Dental Products. Fortress Information Leaflet. Kansas City, Kan, USA. Chameleon Dental Products.

8 Ivoclar-Vivadent. IPS Empress Information Leaflet. Schaan, Liechtenstein: Ivoclar-Vivadent.

9 Burke F J T. The effect of variations in bonding procedure on fracture resistance of dentin-bonded all-ceramic crowns. Quintessence Int 1995; 26: 293-300.

10 Brunton P A, Wilson N H F. Preparations for porcelain laminate veneers in general dental practice. Br Dent J 1998; 184: 553-556.

11 Mayers R E et al. Standards of Human Occlusal Development. Center for Human Growth and Development — The university of Michigan, 1976; 45-48.

12 Etemadi S, Smales R J, Drummond P W, Goodhart J R. Assessment of tooth preparation designs for posterior resin-bonded porcelain restorations. J Oral Rehabilitation 1999; 26: 691-697.

13 Strohaver R A. A comparison of articulator mountings made with centric relation and myocentric position records. J Prosthet Dent 1972; 28: 379-390.

14 Landis J R, Koch G C. The measurement of observer agreement for categorical data. Biometrics 1977; 33: 159-174.

15 Smith P W, Richmond R, McCord J F M. The design and use of special trays in prosthodontics: guidelines to improve clinical effectiveness. Br Dent J 1999; 187: 423-426.

16 Winstanley R B, Carrotte P V, Johnston A. The quality of impressions for crowns and bridges received at commercial dental laboratories. Br Dent J 1997; 184: 209-213.

17 Shillingburg H T, Hobo S, Whitsett L D. Fundamentals of fixed prosthodontics. 3rd ed. Chicago: Quintessence Publishing Co. 1997.

18 Karlsson S, Landah I, Stegersjo G, Milleding P A. A clinical evaluation of porcelain laminate veneers. Int J Prosthodont 1992; 5: 447-451.

19 Lacy A M, Wada C, Du W, Watanabe L. In-vitro microleakage at the gingival margin of porcelain and resin veneers. J Prosthet Dent 1992; 67: 7-10.

20 Sim C, Neo J, Chua E K, Tan B Y. The effect of dentine bonding agents on the microleakage of porcelain veneers. Dent Mater 1994; 10: 278 281

21 Preston J D. Rational approach to tooth for ceramo-metal restorations J Prosthet Dent 1977; 21: 683-698.

22 Saunders W P, Saunders E M. Prevalence of periradicular periodontitis associated with crowned teeth in an adult Scottish subpopulation. Br Dent J 1998; 185: 137-140.

23 Christensen G J. Tooth preparation and pulp degeneration. J Am Dent Assoc 1997; 128: 353-354.

24 Burke F J T. Maximising the fracture resistance of dentine-bonded all-ceramic crowns. J Dent 1999; 27: 169-173.

25 Broderson S P. Complete-crown and partial tooth preparation designs for bonded cast ceramic restorations. Quintessence Int 1994; 25: 535539 .

26 Rimmer S E, Mellor A C. Patient's perceptions of aesthetics and technical qualities of crowns and fixed partial dentures. Quintessence Int 1996; 27: 155-162. 